\title{
Extraction and Electrochemical Fingerprint of Polysaharide and Fat Soluble Compounds from Panax
}

\author{
Xiaohui Wang, Dong Liu, Xia Han, Jinhua Tan, Cheng Qi, Pengfei Chen, \\ Xiaoling Yang*
}

People's Hospital of Bayinguoleng Mongolian Autonomous Prefecture, No.41 Renmin East Road, Korla City, Xinjiang, PR China

*E-mail: ty_wxh@163.com

Received: 28 July 2021 / Accepted: 30 August 2021 / Published: 10 October 2021

\begin{abstract}
Plant systematics focuses on the evolutionary results of organisms at a particular time in the process of evolution, which aims to elucidate the evolutionary processes and patterns as well as the kinship among various taxa. Panax is a medicinally important genus within the family of Araliaceae, in which almost all species are of cultural significance for traditional Chinese medicine. It is of great significance to conduct an in-depth study on the phylogeny of the species of the genus Panax and to introduce their introduction of cultivation and utilization. The application of electrochemical fingerprints in plant phylogenetic study is an emerging research field in related to biosensors. In this work, the lipid-soluble components, ginsenosides and polysaccharides were extracted from Panax trifolius, $P$. stipuleanatus, $P$. pseudoginseng, $P$. notoginseng, $P$. quinquefolius, $P$. japonicas, and $P$. ginseng with supercritical $\mathrm{CO}_{2}$ extraction technique. The electrochemical fingerprints of the 7 species of Panax were recorded with lipid-soluble components and polysaccharides extract. The results indicate that tetraploid species $P$. ginseng and $P$. quinquefolius are allotetraploids. $P$. notoginseng, $P$. ginseng and $P$. quinquefolius are clustered in one large branch and are more closely related. The diploid species $P$. trifolius and the tetraploid $P$. quinquefolius are both distributed in North America, but they are distributed on two different sub-branches on the same phylogenetic branch.
\end{abstract}

Keywords: Electrochemical fingerprint; Electroactive compounds; Panax; Phylogenetic study; Taxonomy

\section{$\underline{\text { FULL TEXT }}$}

(C) 2021 The Authors. Published by ESG (www.electrochemsci.org). This article is an open access article distributed under the terms and conditions of the Creative Commons Attribution license (http://creativecommons.org/licenses/by/4.0/). 